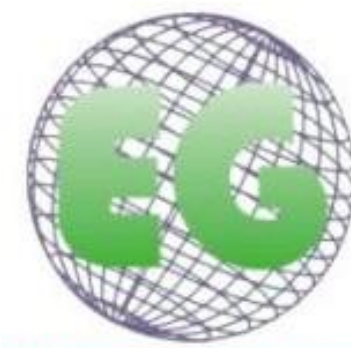

ISSN 1695-6141 $N^{\circ} 51$

\title{
Estudio situacional de los registros electrónicos en una unidad de medicina interna: barreras y oportunidades
}

A situational study of electronic records in an internal medicine unit: barriers and opportunities

\author{
Montserrat Pérez-Martí ${ }^{1}$ \\ Lina Cristina Casadó Marín ${ }^{2}$ \\ José Oriol Romaní Alfonso ${ }^{2}$
}

${ }^{1}$ Diplomada en Enfermería. Máster en Investigación en Ciencias de la Enfermería. Doctoranda de la Universidad Rovira y Virgili. Enfermera Historia Clínica Electrónica y Urgencias. Hospital Comarcal Alt Penedés. Vilafranca del Penedés. Barcelona. España.

${ }^{2}$ Profesor/a de la Universidad Rovira i Virgili.Tarragona. España.

\section{E-mail: montserratperez@csap.cat}

\section{http://dx.doi.org/10.6018/eglobal.17.3.284211}

Recibido 23/02/2017

Aceptado 22/05/2017

\section{RESUMEN:}

El objetivo de este trabajo fue conocer los recursos, flujos de trabajo y percepción de las enfermeras de una unidad de medicina interna, relacionados con los registros electrónicos, con el fin de determinar si la implantación de tablets en las unidades de hospitalización, podrían responder a sus expectativas.

Se llevó a cabo un estudio descriptivo, transversal, muestreo no probabilístico, consecutivo durante los meses de julio y agosto del 2016. Se realizaron 31 observaciones, a 18 enfermeras, 392 pacientes ingresados. Las variables: turno, metodología de trabajo, tiempo "ronda", tiempo transcripción de datos, tiempo total, número de pacientes ingresados y tiempo invertido por paciente. También se preguntó a cada enfermera sobre cómo trabajaban y el motivo.

La media de tiempo total fue 59,16 min (DT:16,6), y 12,65 (DT:1,11) pacientes ingresados. Tiempo invertido por paciente 4,65 min (DT:1,15), inferior en el turno tarde [M:4,14; DT: 0,84] que el de noche [M: 5,47; DT: 1,12]. El turno noche siempre utilizaba el mismo método de trabajo y era el turno tarde que presentaba sistemáticas diferentes. Las enfermeras manifestaron la necesidad de un sistema de registro ligero, en tiempo real, a pie de cama del enfermo, evitando desplazamientos, de fácil acceso para la consulta e intercambio de información.

Los dos métodos de trabajo de la unidad de observación no satisfacen las necesidades de las enfermeras, son lentos, pesados y dificultan el acceso a la información y el registro. La incorporación de tablets podría reducir estos problemas y cubrir sus expectativas, abriendo una nueva línea de investigación.

Palabras clave: Registros Electrónicos de Salud; Enfermería; Computadores de Bolsillo (tablets); Equipamientos y Suministros; Medicina Interna 


\begin{abstract}
:
The objective of this study was to identify the resources, workflows and perception of nurses of internal medicine unit, related to electronic records in order to determine if the implementation of tablets in hospital units could cover the nurses' needs more satisfactorily.

A descriptive, cross-sectional study with non-probability sampling was carried out during July and August of 2016. The study involved 31 observations of 18 nurses, with 392 patients admitted. The variables were: shift, working methodology, "round" time, time taken to write up data, total time, number of patients admitted and time spent per patient. Each nurse was asked how they worked and why.

The average total time was 59,16 min (DT:16,6), and 12.65 (DT:1,11) patients admitted. Time spent per patient was 4,65 min (DT:1,15) and was lower during the afternoon shift [M:4,14; [DT: 0.84] than during the night shift [M: 5.47; DT: 1,12]. The night shift always used the same method of work, whereas different systems were used during the afternoon. The nurses expressed the need for a lightweight record system that would enable them to record information in real time near the patient without having to go elsewhere, and which would make it easy to consult and exchange information.

The two working methods used in the observation unit do not satisfy the needs of nurses, are slow and cumbersome and make it difficult for nurses to access and record information. The adoption of tablets could reduce these problems and meet nurses' expectations and thus opens a new line of research.
\end{abstract}

Keywords: Electronic Health Records; Nursing; Computers Handheld; Equipment and Supplies; Internal medicine

\title{
INTRODUCCIÓN
}

Los registros enfermeros disponen de numerosas finalidades y utilidades para la enfermería, como una evaluación práctica, una fuente de información para la investigación, facilita el progreso escrito de la profesión dejando constancia del acto del cuidado para la docencia/formación, una herramienta para la gestión (cargas de trabajo, evaluación de resultados, asignación de recursos, etc.), legal, de seguridad para los pacientes, de comunicación y continuidad de cuidados. ${ }^{(1-4)}$

No obstante, existe una dificultad en la cumplimentación de los registros por parte de los profesionales de enfermería, en que no acaba de estar toda la información relevante registrada, habiendo notas imprecisas, errores y variabilidad. ${ }^{(1,2,4-6)}$

Por otro lado, los requerimientos institucionales y los cambios tecnológicos han impulsado el desarrollo de los registros electrónicos, que nos han permitido cuantificar y evidenciar la contribución de la enfermería mediante los resultados, mejorar el acceso a la información de forma inmediata y desde cualquier lugar contribuyendo en la toma de decisiones y en la comunicación entre profesionales, facilitan la estandarización de los registros, y la obtención de indicadores que repercute en la seguridad del paciente. ${ }^{(7-11)}$

Pero también nos han traído a desarrollar sistemas fragmentados, lentos, que no facilitan la toma de decisiones, no siendo suficientemente desarrollados, que promueven la transcripción, que dificultan encontrar la información relevante y su cumplimiento, aumentando el tiempo de dedicación de los profesionales. ${ }^{(9-11)}$

Para mejorar los registros, es importante facilitar las herramientas necesarias para mejorar el uso por los profesionales, como son los recursos técnicos que facilitan los registros a pie de cama del enfermo, como las tablets ${ }^{(12-15)}$

La mejora del uso del sistema de registro tiene una repercusión directa en la mejora de la satisfacción de los profesionales ${ }^{(16)}$ y en la seguridad de los pacientes ${ }^{(10,14,17)}$. Pero antes de realizar un cambio de registro con las tablets, se consideró importante conocer cuáles son las necesidades de los profesionales, sus expectativas, y los 
sistemas de trabajo. Con esta información, pudimos valorar su idoneidad, cuáles podían ser los beneficios esperados. Es decir, dispusimos de una aproximación a la realidad de una unidad de medicina interna de un hospital comarcal y de los sistemas de registros existentes.

\section{OBJETIVOS}

Conocer los recursos de la unidad de trabajo que están relacionadas con los registros. Determinar los flujos de trabajo: tiempo de "ronda", tiempo de transcripción de datos y tiempo total.

Conocer la percepción de la enfermera sobre el sistema de registro.

Conocer las expectativas de las enfermeras de un sistema de registro y si las tablets podrían responder a sus expectativas.

\section{METODOLOGÍA}

Se llevó a cabo un estudio descriptivo, transversal durante los meses de julio y agosto de 2016. La población de estudio fueron todas aquellas enfermeras que estuvieran trabajando en una unidad de medicina interna. Los criterios de inclusión: que estuvieran contratadas, con un nivel de experiencia de 3 años y que quisieran participar voluntariamente ${ }^{(18)}$. Los criterios de exclusión: que estuvieran en situación de baja en el momento de la recogida de datos.

Se llevó a cabo un muestreo no probabilístico, consecutivo, todas las enfermeras que cumplían con los criterios de inclusión en el periodo de estudio. Es decir, durante el periodo de recogida de datos las que estaban trabajando en aquel momento que cumplían con los criterios de inclusión. Por lo tanto, no se observaron personas, sino oportunidades. Una persona pudo tener más de una observación u oportunidad. Una misma persona siempre fue observada en el mismo turno de trabajo.

Y se observó este momento nombrado tiempo "ronda", la rutina establecida que se realiza al principio del turno de tarde y noche, donde se realiza la toma de constantes y las actividades enfermeras estandarizadas.

Las enfermeras disponían de ordenadores portátiles fijados a los carros de unidosis ubicados, la mayor parte del tiempo, a la zona de trabajo. Para realizar la "ronda", según criterio de la enfermera, utilizaba dos métodos diferentes. El primero, llamado método separado, consulta de la Historia Clínica con el ordenador en la zona de trabajo, medida de constantes y registro en un papel, transcripción posterior. El segundo, llamado método junto, la enfermera salía de la zona de trabajo al pasillo con un carro con portátil, consulta, medida dentro de la habitación, y registro desde la puerta de entrada de la habitación. El carro con el portátil no podía entrar en la habitación. En ningún caso se podía hacer consulta, medida y registro a pies de cama del enfermo. La "ronda" no se realizaba en turno de mañana.

Se realizaron 31 observaciones a 18 enfermeras, 392 pacientes ingresados. Cada observación se mantuvo en el inicio del turno de trabajo y tuvo una duración máxima de 3 horas. 
Los instrumentos para la recogida de la información fueron una base de datos con las variables de estudio. Se definieron variables estructurales como turno, método, tiempo "ronda", tiempo transcripción de datos, tiempo total, número de pacientes ingresados y tiempo invertido por paciente. Se identificó como variable principal el tiempo invertido por paciente, debido a que esta es más estandarizada, teniendo en cuenta los pacientes ingresados.

La selección de las variables está justificada en un estudio realizado en un hospital de Toronto. En que se medían los tiempos de registros, el tiempo total, el tiempo invertido por paciente y el número de pacientes ingresados. En este estudio se comparaba un grupo de registro en papel con otro con registros electrónicos. El resto de variables han sido escogidas para adaptar la investigación al campo de estudio, a la situación práctica y real de las unidades de trabajo y a una composición diferente de los grupos control y experimental (9).

Los datos se analizaron mediante paquete estadístico SPSS 17.0, calculando medidas de tendencia central y dispersión. Para comprobar la normalidad de las variables se utilizó la prueba de Shapiro-Wilk y se aplicaron pruebas paramétricas y no paramétricas (Exacto de Fisher, T de Student).

También se preguntó, en forma de conversación informal, a cada enfermera sobre cómo trabajaban y el motivo, ¿qué método utilizas?, ¿por qué motivo?. Las respuestas se agruparon, elaborando ejes temáticos y categorías.

El presente trabajo forma parte del transcurso de investigación de una tesis doctoral con informe favorable del Comité Ético de Investigación Clínica de Bellvitge. Se han respetado la intimidad y confidencialidad de los informantes.

\section{RESULTADOS}

Del total de la muestra $(n=31)$, un $61,3 \%(n=19)$ de las observaciones se realizaron en el turno de tarde y un $38,7 \%$ en el de noche $(n=12)$.

Si valoramos el método de trabajo, el sistema junto es más representativo, con un $74,2 \%$, y el sistema separado con un $25,8 \%$. Por lo que podemos decir, que la mayoría de los profesionales prefieren salir con el carro con el portátil al pasillo y realizar todas las actividades a la vez (ver tabla 1 ).

Tabla 1. Variables categóricas

\begin{tabular}{|c|c|c|}
\hline & $n=31$ & $\%$ \\
\hline \multicolumn{3}{|l|}{ Turno } \\
\hline Mañana & 0 & 0 \\
\hline Tarde & 19 & 61,3 \\
\hline Noche & 12 & 38,7 \\
\hline \multicolumn{3}{|l|}{ Método } \\
\hline Junto & 23 & 74,2 \\
\hline Separado & 8 & 25,8 \\
\hline
\end{tabular}

Elaboración propia 
Al relacionar turno de trabajo y método obtuvimos que un $100 \%$ del turno de noche utiliza el método junto. El método separado sólo se observó en el turno de tarde. Un $57,9 \%$ de profesionales de este turno utilizan el método junto y un $42,1 \%$ separado $(p=0,0012)$ (ver tabla 2).

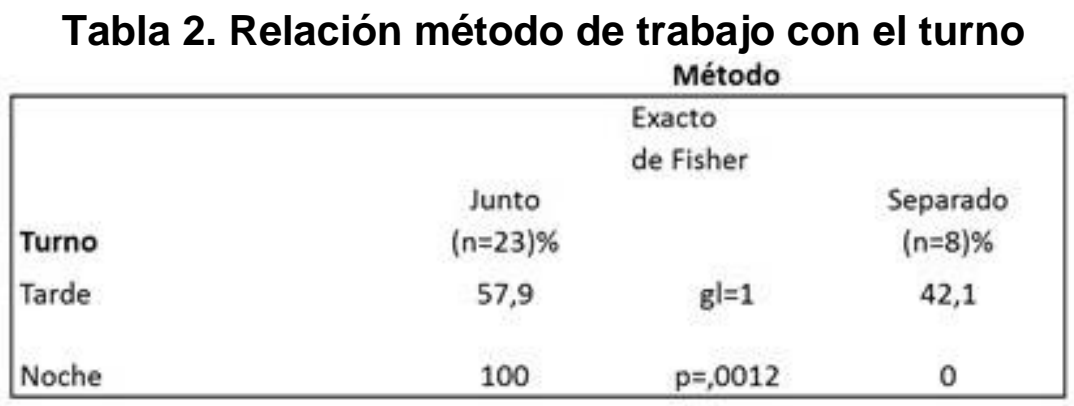

Elaboración propia. * $\mathrm{p}<, 05$

De las variables cuantitativas, se constató una media de 12,65 [DT: 1,11] pacientes ingresados durante el periodo de estudio.

Y del tiempo de dedicación de los profesionales de enfermería a realizar la "ronda", una media de 35,88 [DT: 10,5] minutos, tiempo de transcripción de datos de 12,63 [DT: 1,77] minutos y el tiempo total 59,16 [DT: 16,6] min y un tiempo invertido por paciente de 4,65 [DT: 1,15] minutos (ver tabla 3).

Tabla 3. Variables cuantitativas

\begin{tabular}{|lrr|} 
& \multicolumn{2}{c|}{ Total $(\mathbf{n}=31)$} \\
\hline M & 35,88 & 10,5 \\
\hline Tiempo Ronda & 12,63 & 1,77 \\
Tiempo Traspaso datos & 59,16 & 16,6 \\
Tiempo Total & & \\
No Pacientes Ingresados & 12,65 & 1,11 \\
Tiempo Invertido Paciente & 4,65 & 1,15 \\
\hline
\end{tabular}

Elaboración propia

Seguidamente, se procedió a analizar la variable principal, el tiempo invertido por paciente, con el resto de las variables categóricas.

La prueba de normalidad Shapiro-Wilk (S-W) por $n<50$, nos indica que no se puede rechazar la hipótesis $\mathrm{H}_{0}(\mathrm{p}<0,05)$, y que el tiempo invertido por paciente que dedican los profesionales es uniforme, sigue una distribución normal en los dos turnos de trabajo (S-W noche=, 154 ; tarde $=, 161)$.

Al valorar el tiempo invertido por paciente entre los diferentes turnos para muestras independientes (una misma persona sólo se observó en un único turno), se obtuvo que el tiempo invertido por paciente del turno de noche [M: 5,47; DT: 1,12] fue superior al de tarde [M: 4,14: DT: 0,84]. Existiendo diferencias significativas entre las medias con varianzas similares $(F$ de Levene $=0,44 p=0,511$; T Student: $-3,76 p=0,001$ ). 
En cambio, de esta misma variable, no se obtuvieron diferencias entre las medias del método junto [M: 4,41: DT: 0,71] y separado [M: 3,76: DT: 0,91] (F de Levene =1,107 $p=0,307$; T Student: 1,$73 ; p=0,101)$.

En otras palabras, se concluyó que el método de trabajo utilizado por los profesionales de enfermería no influye significativamente con el tiempo invertido por paciente. Pero sí que existen diferencias entre turnos (ver tabla 4)

Tabla 4. Relación tiempo invertido por paciente con el turno y método:

\begin{tabular}{|c|c|c|c|c|}
\hline & \multicolumn{2}{|c|}{ Turno $(n=31)$} & \multicolumn{2}{|c|}{ Método ( $n=31$ ) } \\
\hline & $\begin{array}{l}\text { Tarde } \\
(n=19)\end{array}$ & $\begin{array}{l}\text { Noche } \\
(n=12)\end{array}$ & $\begin{array}{l}\text { Junto } \\
(n=23)\end{array}$ & $\begin{array}{l}\text { Separado } \\
\quad(n=8)\end{array}$ \\
\hline M & 4,14 & 5,47 & 4,41 & 3,76 \\
\hline DT & 0,84 & 1,12 & 0,71 & 0,91 \\
\hline$T / U$ & \multicolumn{2}{|c|}{$\begin{array}{c}T / U:-3,76(P=, 001) \\
g \mid=29\end{array}$} & \multicolumn{2}{|c|}{$\begin{array}{c}T / U=1,73(p=, 101) \\
g l=29\end{array}$} \\
\hline
\end{tabular}

Elaboración propia. * $\mathrm{p}<, 05 \mathrm{~T} / \mathrm{U}=\mathrm{T}$ de Student

Curiosamente, algunos de los informantes, en su discurso referente a la pregunta sobre el motivo de utilizar un sistema de trabajo u otro, tanto el grupo junto como separado pensaban lo mismo, que ahorran tiempo.

El grupo junto, el ahorro se derivó de la percepción que al poder realizar las actividades todas juntas (constantes, vías, medicación, etc.), a la vez, se necesita menos tiempo. $Y$ que también se ahorraban desplazamientos por el pasillo, que después podían invertir en otras actividades.

"Ho faig tot en el moment i me'n oblido. Hem fa l'efecte que estalvio temps" INF01

"És més còmode, administro la medicació de les 16h, faig menys viatges i puc consultar qualsevol dubte que tinc" INF09

En cambio, el grupo separado, argumentaba el ahorro de tiempo en el hecho de poder ir con el aparato eléctrico de constantes y el papel les permitía ser más ágiles, moverse con más rapidez porque llevaban menos peso, también les permitía poder entrar dentro de las habitaciones con los aparatos, es decir, estar a pie de cama del paciente.

"porque tengo la impresión de que voy más rápida... No entro y salgo tanto de la habitación..."INF05

"És més pràctic i àgil, en quan a temps i en quan a portar menys recursos a sobre, el carro pesa molt..." INF07

"Un dels motius es que no he de CARREGAR amb el carro de les unidosis que portar al damunt l'ordinador, pesa un mort i les rodes giren fatal"! INF08. 
Los profesionales articularon como una fuente de dificultad el disponer de un carro muy pesado y de difícil manejo.

Del discurso de los informantes del turno de noche en que explicaban los motivos de utilizar el método de trabajo junto se complementaron estos resultados.

Para este equipo, era relevante poder hacer todas las actividades todas juntas para evitar entrar y salir de las habitaciones en diferentes horas y despertar a los pacientes, favoreciendo el descanso nocturno y su bienestar.

"És per a confort del pacient, per entrar i sortir menys de l'habitació. D'aquesta manera ho fas tot junt, mires les constants..., la medicació i molestes menys " INF12 i 13.

También manifestó como una dificultad que hubiera una auxiliar menos y que dependieran de ayudas puntuales, motivos de las diferencias de tiempos.

Favorecer el descanso nocturno de las personas ingresadas, fue una intervención enfermera prioritaria del personal del turno por la noche, que prevaleció por delante del ahorro de tiempo, y uno de los motivos de escoger el método a la vez.

El turno por la noche, también mencionaron como significativo, la seguridad del paciente. Uno de los motivos que postulaban para utilizar el método junto fue evitar errores en la transcripción de datos.

"Por separado hay más posibilidades de error, así es más practico" INF18.

\section{DISCUSIÓN}

De los resultados obtenidos se evidenció que el turno de noche siempre utilizaba el método junto y que era el turno de tarde el que presentaba diferentes sistemáticas. La incorporación de tablets podría unificar sistemas de trabajo.

Uno de los motivos de los informantes para justificar la elección de un método de trabajo, fue el ahorro de tiempo. En cambio, del análisis de los datos cuantitativos no se encontró relación entre estas dos variables.

Para las enfermeras el tiempo es un concepto importante y basan su manera de organizarse en función de poder realizar las actividades de enfermería de una forma rápida. Enfermería es una profesión pragmática, en que se valoran los resultados reales y prácticos de las acciones efectuadas. El ahorro de tiempo en actividades burocráticas es un concepto presente y, por tanto, importante. Por lo que antes de implementar cualquier cambio en los sistemas de trabajo se tendría que tener muy presente esta categoría, si se quiere que sea aceptado por los profesionales.

En la bibliografía referenciada se constata que aquellas experiencias que comportan una disminución de actividades burocráticas o administrativas, y que evitan realizar registros duplicados, son percibidas positivamente por las enfermeras. También se perciben como agentes facilitadores sistemas que permitan el rápido y fácil acceso a la información necesaria y la comunicación. Estableciéndose una vinculación inherente de estas consideraciones con el ahorro de tiempo. Esta reducción puede ser invertida en tiempo de dedicación y atención directa al paciente [11,14,15,19, 20]. 
No obstante, la implantación de cualquier recurso o cambio tecnológico tiene que ser realizado teniendo en cuenta los flujos de trabajo y facilitando la participación de los profesionales para adelantarse a las dificultades y adecuarlo a las necesidades $[12,13,16]$.

Existen estudios que describen experiencias infructuosas en relación a los registros electrónicos y/o recursos, generando frustraciones y resistencias al cambio [20, 21, 22].

Se constató una media 12,65 pacientes, un tiempo total 59,16 min y un tiempo invertido por paciente de 4,65 minutos. Los resultados de un estudio similar realizado en un hospital de Toronto fueron, 4 o 6 pacientes, tiempo total de 65,2 min, tiempo invertido por paciente de 13,04 min con registros electrónicos [12]. Al comparar los resultados se evidencia, que la ratio de la unidad de este estudio es el doble y los tiempos inferiores.

No se encontraron estudios similares realizados en el estado español, por lo que no nos pudimos comparar con otros hospitales en que exista una realidad del campo de estudio similar. No obstante, con la obtención de estos resultados sí que nos podremos comparar con nosotros mismos.

Sí se evidenció una diferencia de tiempo entre los turnos de trabajo, el tiempo invertido por paciente del turno de noche fue 1,33 min superior. Por lo que en el momento de evaluar cualquier modificación en los flujos de trabajo se tendría que hacer por cada uno de estos grupos de forma independiente.

\section{Limitaciones del estudio}

Ha habido limitaciones en la captación de la muestra. Al obtener la muestra por oportunidades $u$ observaciones, se ha observado diferentes veces a un mismo individuo, teniendo poco control en las diferencias de comportamiento individuales y en las repeticiones de las observaciones. Se considera que para próximos estudios sería necesario establecer un grupo determinado de participantes en cada turno con el mismo número de observaciones.

\section{CONCLUSIONES}

Los dos métodos de trabajo de la unidad de observación no satisfacen las necesidades de las enfermeras, son lentos, pesados y dificultan el acceso a la información y el registro. Son sistemas antiguos, mal desarrollados.

Sería imprescindible actualizarlos, renovarlos, incorporando nuevas herramientas, como las tablets o programas que favorezcan la interoperabilidad semántica entre sistemas.

Con las tablets, se unificarían metodologías entre el turno de tarde y el de noche, se dispondría de un sistema ligero, en que se podría trabajar en tiempo real a pie de la cama del enfermo, mejoraría los flujos de trabajo, el acceso a la información, la comunicación entre profesionales, la seguridad y bienestar del paciente, la calidad de los registros y la satisfacción de los profesionales.

Con los resultados pudimos construir una hipótesis de trabajo, que los registros a pie de la cama del enfermo con tablets disminuyen los tiempos de registros y aumentan la 
satisfacción de los profesionales. Como no se obtuvo resultados similares a ningún hospital en que nos pudiéramos comparar, se abriría una nueva línea de investigación

\section{REFERENCIAS}

1. Calero Romero MR, González FL. El registro de enfermería como parte del cuidado, En: euroenfermería ante la patología tumoral y degenerativa. XXXVIII Congreso Nacional de la Asociación Española de Enfermería en neurociencias; 2014; Granada, España.

2. Ortiz C O, Chávez M. El registro de enfermería como parte del cuidado. Scientia 2006;8 (2): $52-6$

3. Currell $R$, Urquhart $C$ Sistemas de registro de enfermería: efectos sobre la práctica de la enfermería y resultados en la asistencia sanitaria (Revisión Cochrane traducida). En: La Biblioteca Cochrane Plus, 2008 Número 4. Oxford: Update Software Ltd. [citado 12 abr 2016] Disponible en: http://www.bibliotecacochrane.com/BCPGetDocument.asp?SessionID=\%20115142 51\&DocumentID=CD002099

4. Saranto k, Ulla Mari K. Evaluating nursing documentation-research desings and methods: systemtic review. J Adv Nurs 2009; 65(3):464-76

5. Amezcua M. La calidad en la documentación clínica de enfermería. Del papeleo a los registros para el cuidado. Index Enferm (edición digital) 1995; 11.

6. Del Olmo SM, Casas L, Mejías A. En registro de enfermería, un sistema de comunicación. Enferm Clínica 2007, 17 (3):142-145.

7. Consejo de la Profesión Enfermera de Cataluña. Indicadores de Avaluación de los Cuidados Enfermeros. Generalidad de Cataluña. Departamento de Salud. Barcelona, enero 2014. [citado 12 abr 2016]: Disponible en: http://coibazure.blob.core.windows.net/coib-publish/invar/a840d315-8fa6-4d51ab3e-819863b23710

8. Seguridad de Pacientes. [Internet]. Barcelona. Generalidad de Cataluña. [citado 20 abr 2016]: Disponible en:

http://seguretatdelspacients.gencat.cat/ca/professionals/projectes de seguretat d els pacients/

9. Medeiros AL, Rezende LCM, Santos SR, Nascimento NM, Costa T. Applicability of electronic medical records in health sector: an integrative review. J Nurs UFPE on line 2014; 8 (11): 3992-4000.

10. Fernández M, Cibanal JL. Los sistemas de Información Guiados por el Conocimiento. El futuro de los Registros Enfermeros. Rev Tesela 2009; 5.

11. Kelley F K, Drandon D H. Electronic Nursing Documentation as a Strategy to Improve Quality of Patient Care. J Nurs Scholarsh, 2011; 43:2, 154-162.

12. Yeung M S, Lapinsky S E, Granton J T, Doran D M, Cafazzo J A. Examining nursing vital signs documentation workflow: barriers and opportunities in general internal medicine units. J Clin Nurs. 2012 Apr;21(7-8):975-82. doi: 10.1111/j.13652702.2011.03937.x. Epub 2012 Jan 14.

13. Sockolow PS, Rogers M, Bowles KH, Hand KE, George J. Challenges and facilitators to nurse use of a guideline-based nursing information system: Recommendations for nurse executives. Appl Nurs Res 2014; 27: 25-32.

14. Fleischmann R; Duhm J, Hupperts Hagen, Brandt SA. Tablet computers with mobile electronic medical records enhance clinical routine and promote bedside time: a controlled prospective crossover study. J Neurol 2015; 262: 532-540.

15. Stuttle B. Computers in nursing: patient data at the point of care. Prim Health Care 2009; 19 (5); 32-33. 
16. Dykes PC, Carroll DL, Benoit A, et al. A Randomized Trial of Standardized Nursing Patient Assessment Using Wireless Devices. J Am Med Inform Assoc Annual Symposium Proceedings. 2007;2007:206-210

17. Ferreira PA, Marcon, GT; Carvalho DC. Contribuições dos registros eletrônicos para a segurança do paciente em terapia intensiva: uma revisão integrativa. Texto Contexto Enferm | 2012 | 21(4):971-979. [citado 15 oct 2015] Disponible en: http://www.index-f.com/textocontexto/2012pdf/21-971.pdf.

18. Benner PE. From novice to expert: excellence and power in clinical nursing practice. Menlo Park (CA): Addison-Wesley; 1984

19. Blake $\mathrm{H}$. Mobile technology: streamlining practice and improving care. $\mathrm{Br} \mathrm{J}$ Community Nurs 2013; 18 (9).

20. Gregory D, Buckner M. Poibt-of-Care Technology. Integration for Improved Delivery of Care. Crit Care Nurs Q 2014; 37 (3); 268-272

21. Haller G, Haller DM, Courvoisier DS, Lovis C. Handheld vs. Laptop Computers for Electronic Data Collection in Clinical Research: A Crossover Randomized Trial. J Am Med Inform Assoc 2009; 16 (5).

22. Kohle A, Chatterjee $P$, Ulku H, Hochhheiser H, Bartos $\mathrm{C}$. Evaluating the Barriers to Point-of-Care Documentation for Nursing Staff. Comput Inform Nurs 2012; 30 (3); 126-133 